\title{
Straightforward and accurate technique for post-coupler stabilization in drift tube linac structures
}

\author{
Mohammad Reza Khalvati ${ }^{1,2, *}$ and Suitbert Ramberger ${ }^{2, \dagger}$ \\ ${ }^{1}$ School of Particles and Accelerators, Institute for Research in Fundamental Sciences (IPM), \\ P.O. Box 19395-5531, Tehran, Iran \\ ${ }^{2}$ CERN, 1211 Geneva 23, Switzerland \\ (Received 18 December 2015; published 12 April 2016)
}

\begin{abstract}
The axial electric field of Alvarez drift tube linacs (DTLs) is known to be susceptible to variations due to static and dynamic effects like manufacturing tolerances and beam loading. Post-couplers are used to stabilize the accelerating fields of DTLs against tuning errors. Tilt sensitivity and its slope have been introduced as measures for the stability right from the invention of post-couplers but since then the actual stabilization has mostly been done by tedious iteration. In the present article, the local tilt-sensitivity slope $\mathrm{TS}_{n}^{\prime}$ is established as the principal measure for stabilization instead of tilt sensitivity or some visual slope, and its significance is developed on the basis of an equivalent-circuit diagram of the DTL. Experimental and 3D simulation results are used to analyze its behavior and to define a technique for stabilization that allows finding the best post-coupler settings with just four tilt-sensitivity measurements. CERN's Linac4 DTL Tank 2 and Tank 3 have been stabilized successfully using this technique. The final tilt-sensitivity error has been reduced from $\pm 100 \% / \mathrm{MHz}$ down to $\pm 3 \% / \mathrm{MHz}$ for Tank 2 and down to $\pm 1 \% / \mathrm{MHz}$ for Tank 3. Finally, an accurate procedure for tuning the structure using slug tuners is discussed.
\end{abstract}

DOI: 10.1103/PhysRevAccelBeams.19.042001

\section{INTRODUCTION}

In manufacturing and operation of long accelerating structures, stabilization and tuning is essential as various factors such as manufacturing tolerances, movable tuner movement, and beam loading contribute to tuning errors. Since drift tube linacs (DTLs) operate in the $\mathrm{TM}_{010}$ accelerating mode which corresponds to the 0 -mode in a nonstabilized cavity, the sensitivity of axial fields to tuning errors is critical. In 1967, based on their work on coupled cavity chains, Knapp, Swenson, and Potter, invent a resonant coupling device that they call post-coupler to minimize the sensitivity of the $\mathrm{TM}_{010}$ axial electric field mode to tuning errors [1]. When correctly tuned, the postcouplers have their modal spectrum just below that of the accelerating modes. At this point they are in confluence and make the cavity operate in a hybrid $\pi / 2$-type mode. In their paper that was published just after the filing of the patent, global tilt-sensitivity measurements, the closing of the stop band, and the importance of tilt-sensitivity slope for improving local stability are discussed however without providing a straightforward procedure for stabilization [2].

\footnotetext{
${ }^{*}$ Khalvati@ipm.ir

†Suitbert.Ramberger@cern.ch
}

Published by the American Physical Society under the terms of the Creative Commons Attribution 3.0 License. Further distribution of this work must maintain attribution to the author(s) and the published article's title, journal citation, and DOI.
In order to make finding the starting point for the postcoupler adjustment near confluence easier, a distortion parameter was introduced [3]. It determines how much tuning error deforms the electric fields over the whole structure, and it is defined as the sum of the absolute differences in each cell between the field levels with the post-coupler set to length $x$ and either the cavity without post-couplers $D_{x}=\sum_{i}\left|E_{0 i}-E_{0 i}^{n}\right|[3,4]$ or the average field $D_{x}=\sum_{i}\left|E_{0 i}-\bar{E}_{0}\right|[5,6]$. All post-couplers are set at the same length $x$. When one of the post-coupler modes resonates close or at the frequency of the accelerating mode, strong interaction leads to high distortion parameter values $D_{x}$. Except for [3] that uses traditional tilt-sensitivity measurements for final stabilization, the minimum of the distortion parameter is used as the stabilization point without further explanation. In [6] individual resonance frequencies of the post-couplers are measured, in order to find a variation of post-coupler lengths along the cavity.

A further approach to stabilization has been described in [7]. Two criteria are required; (a) the frequency shifts of the upper and lower modes nearest the operating mode shall be equal, and (b) the electric field distributions of the two neighboring modes along the cavity shall be similar. A variant to this approach is discussed in [8] with the first criterion formulated as (a) close the stop band between the dispersion curve of the post-mode and that of the TMmode. While closing the stop band is a basic requirement in order to get the stabilization effect of a $\pi / 2$ mode structure, the condition for equal frequency shifts is fulfilled only roughly. This comes as no surprise if one considers that the 
coupling between the main resonators is much stronger than their coupling with the system of post-couplers. In consequence, the mode separation between the $\mathrm{TM}_{01 x}$ modes is much larger than the mode separation between the post-coupler modes. Electric field distributions of the two neighboring modes have some resemblance when cavities are stabilized to flat tilt sensitivity (e.g. [8,9]), however there is no straightforward procedure to adjust post-couplers other than by tedious iteration, nor is there a proof for the argument that the field distributions need to be similar.

Notwithstanding the many attempts to find a better technique, the classic tilt-sensitivity approach has been used in numerous applications. A peculiar case is a rampedgradient DTL [10]. The oft-cited paper describes not only the tilt-sensitivity measurement with its convenient unit of $\% / \mathrm{MHz}$ which takes account of the proportionality of the tilt sensitivity to the amount of perturbation, but also how to reach an accuracy at the percentage level in beadperturbation measurements as is required in the tuning of long DTL structures. The tuning is undertaken by moving post-couplers individually in order to reach low local tilt-sensitivity slope and orienting tabs on each postcoupler tip for cell tuning. Complementary information can be drawn from [11], where post-couplers are moved in unison and the tilt sensitivity is analyzed in relation to the post-coupler length. The strong interaction of the accelerating mode with the post-coupler mode is visible as a pole in the plots. This result explains the bumps in the distortion parameter curves which are an artifact of taking the absolute value in the respective formula. However with the recipes of these papers and publications like [12], there is still no straightforward procedure that could limit the stabilization effort to a predictable number of lengthy bead-perturbation measurements.

In order to better understand the cavity behavior, equivalent-circuit models have been derived and its parameters extracted [5,13-16]. Two resonance conditions are discussed in [5] with zero and infinite coupling admittance that will be rediscussed in this paper. It is shown that zero admittance leads to a stabilized field distribution. The same stabilization point is found in [16] by a transport matrix notation. There the numerical model is fitted to modal measurements by adjusting the post-coupler parameters. The post-coupler spectrum fits the measurements only when mutual inductances are taken into account. The variation of post-coupler lengths over the tank is found empirically. While the modal approach provides valuable information, it was felt that the analysis in the circuit "domain" could provide complementary information on stabilization in particular when a local variation of a single post-coupler length along the DTL cavity is to be analyzed. Certainly, the equivalent circuit is only as good as it fits the modal spectrum and its dependencies.

In the present article, a new straightforward and accurate technique to find optimum post-coupler lengths is discussed. It is based on a combination of results of earlier publications in that it (a) analyzes tilt-sensitivity slope $[2,10]$, (b) using an equivalent-circuit model $[13,16]$, and (c) focusing on the conditions of resonance on the postcoupler $[5,16]$. The extraction of the optimum point (d) uses the resonance investigated in [11] however (e) for each post-coupler individually [6].

The derivation of the technique goes beyond previous approaches in that it (a) establishes the local tilt-sensitivity slope $\mathrm{TS}_{n}^{\prime}$ as the principal measure for stabilization, (b) shows that this measure is proportional to the coupling admittance of post-couplers in the equivalent-circuit model, (c) describes a method to extract the individual post-coupler behavior, (d) strictly applies the condition of resonance to the circuit elements of an individual post-coupler for stabilization, and most importantly (e) shows that all the post-couplers of a structure can be adjusted to their individual optimum by only four tilt-sensitivity measurements. Note that even though the technique uses the equivalent-circuit model for its development, it does not require the extraction of all the equivalent-circuit elements.

The current paper thus overcomes the traditional stabilization approach by tedious iteration, where each step requires an individual tilt-sensitivity measurement, and where good stabilization levels typically require several tens of measurements, and replaces it with a carefully derived, straightforward and accurate stabilization technique that can be applied to DTL structures with any number of post-couplers with a limited predefined number of four tilt-sensitivity measurements. In addition and in complement to the stabilization procedure, the optimum setting of tuner lengths is determined by tuning sensitivity measurements. The application of the procedures is demonstrated by the stabilization and tuning of Tank 2 and Tank 3 of the CERN Linac4 DTL. It is considered that the new stabilization technique lends itself also to numerical simulations that allow for the calculation of postcoupler lengths in advance, which will thus drive down costs and complexity of DTL assembly.

In Sec. II of the present paper, the equivalent-circuit model is discussed, in Sec. III the tilt-sensitivity slope between cells $\mathrm{TS}_{n}^{\prime}$ is derived as a function of the postcoupler length. In Sec. IV, it is shown that this measure behaves as predicted from the circuit model. In Sec. V measurements of $\mathrm{TS}_{n}^{\prime}$ of a single post-coupler are compared with simulations, and their dependency on other postcouplers is analyzed. Practical procedures for stabilization and tuning are described in Secs. VI and VII and the stabilization and tuning results on CERN Linac4 DTL cavities are discussed in Sec. VIII.

\section{THE EQUIVALENT-CIRCUIT REPRESENTATION}

DTLs are designed as a series of individual accelerating cells such that each cell resonates at the target operating 
frequency $\omega_{o p}$, while their length increases with the particle velocity $\beta_{n}$ and thus with the particle energy. In the design, the complete structure is assembled from these individual cells that are acting as a strongly coupled resonator chain [17]. In order to study how the accelerating field reacts to local tuning errors, the DTL is modeled as a system of equivalent lumped circuit elements. While it has been shown that the higher resonance frequencies of the different modal systems can only be fitted with more complex implementations [16], for the sake of simplicity, mutual inductances have been neglected as their implementation is neither required for the understanding, nor for the derivation of the stabilization procedure.

The equivalent-circuit representation of an individual accelerating cell equipped with post-couplers is shown in Fig. 1 [13]. Here the $C_{n}$ and $L_{n}$ are the equivalent capacitance and inductance of the $n$th rotationally symmetric resonator cell without post-couplers or stems. They are extracted from the stored energy at the reference voltage, and the resonance frequency. $C_{n}^{\prime}$ is the coupling capacitance between the $n$th drift tube and the cavity wall. Its value can be extracted from tilt-sensitivity measurements or simulations of a cavity without postcouplers. $L_{n}^{\prime}$ mainly represents the inductance of the supporting stem. As was pointed out in [16] for considerations of the accelerating mode, the stem inductance could be neglected as the system of stem resonators in single stem DTLs resonates at comparably low frequencies and is usually not excited at the operating frequency. The post-coupler is about a quarter of a free-space wavelength long and thus close to resonance. It is represented by an inductor $L_{n}^{\prime \prime}$ and a capacitance $C_{n}^{\prime \prime}$ that also contains the capacitance between the $n$th drift tube and postcoupler tip. The structure is considered lossless which is justified by the high quality factors.

As is shown in Fig. 1, the circuit representation of each accelerating cell can be written in terms of an equivalent impedance $Z_{n}\left(\omega, L_{n}, C_{n}\right)$ and a coupling admittance of $Y_{n}^{\prime}\left(\omega, C_{n}^{\prime}, L_{n}^{\prime}, L_{n}^{\prime \prime}, C_{n}^{\prime \prime}\right)$. Here $C_{n}, L_{n}, C_{n}^{\prime}$ and $L_{n}^{\prime}$ depend on the structure geometry, drift tube shape, and gap length. $L_{n}^{\prime \prime}$ and $C_{n}^{\prime \prime}$ depend on the post-coupler length $l_{n}^{\prime \prime}$.

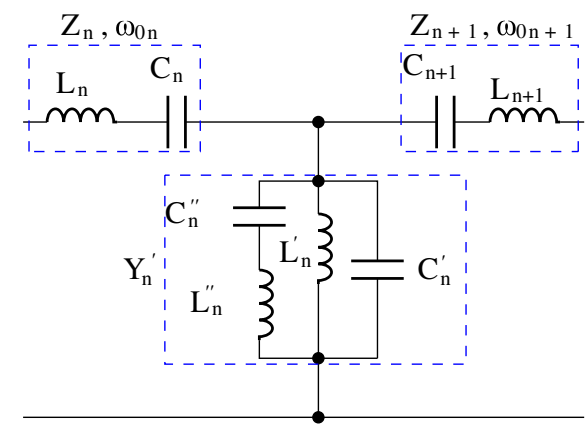

FIG. 1. Equivalent lumped circuit representation of a single DTL cell.
The cell impedance and coupling admittance of the $n^{\text {th }}$ cell are

$$
\begin{aligned}
& Z_{n}=j \omega L_{n}+\frac{1}{j \omega C_{n}}, \\
& Y_{n}^{\prime}=j \omega C_{n}^{\prime}+\frac{1}{j \omega L_{n}^{\prime}}+\frac{j \omega C_{n}^{\prime \prime}}{1-\omega^{2} L_{n}^{\prime \prime} C_{n}^{\prime \prime}} .
\end{aligned}
$$

Applying Kirchhoff's voltage law to the equivalent circuit, that is terminated by a short circuit at either end, the following set of equations can be derived (Fig. 2):

$$
\begin{gathered}
Z_{1} I_{1}+\frac{I_{1}-I_{2}}{Y_{1}^{\prime}}=0, \\
\vdots \\
Z_{n} I_{n}+\frac{I_{n}-I_{n+1}}{Y_{n}^{\prime}}-\frac{I_{n-1}-I_{n}}{Y_{n-1}^{\prime}}=0, \\
Z_{n+1} I_{n+1}+\frac{I_{n+1}-I_{n+2}}{Y_{n+1}^{\prime}}-\frac{I_{n}-I_{n+1}}{Y_{n}^{\prime}}=0, \\
\vdots \\
Z_{N} I_{N}+\frac{I_{N-1}-I_{N}}{Y_{N-1}^{\prime}}=0 .
\end{gathered}
$$

Without driving terms, the system of equations can be rearranged as a generalized eigenvalue system with the resonance frequencies as eigenvalues and the sets of branch currents as corresponding eigenvectors. As the system matrix is sparse with elements around the main diagonal, at a known resonance frequency, the branch currents $I_{n}$ can be solved recursively from either end.

In absence of tuning errors, all cell resonance frequencies $\omega_{0 n}=1 / \sqrt{L_{n} C_{n}}=\omega_{\mathrm{op}}$, and the accelerating mode $\mathrm{TM}_{010}$ resonates at the same frequency $\omega_{\text {op }}$. The cell impedances $Z_{n}$ at resonance are zero, and without perturbation, there is no net voltage on the coupling elements $Y_{n}^{\prime}$. In consequence, post-couplers have no effect on the accelerating field of an unperturbed cavity.

Tuning errors on the other hand influence the intrinsic resonance frequency $\omega_{0 n}$ of an accelerating cell and this leads to nonzero cell impedance at resonance. In a cavity where all the $Y_{n}^{\prime}$ elements were $Y_{n}^{\prime}=0$, the net voltage from detuned cells would lead to a current that continues to

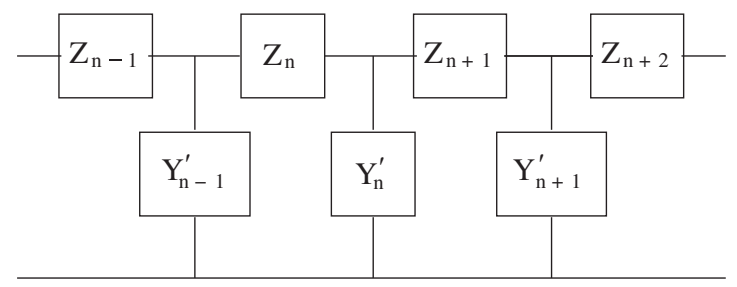

FIG. 2. The schematic representation of three adjacent cells in terms of equivalent impedance and admittance. 
flow through all $Z_{n}$ along the cavity and the effect of a single tuning error would not be detected on neighboring cells.

Real world cavities however have nonzero $C_{n}^{\prime}$ elements. Currents flow on the $Y_{n}$ elements and lead to a voltage variation on the main branches-commonly known as a field tilt- that is decreasing all along the structure from the point of the perturbed cell. The feat of post-couplers now is to introduce a resonant circuit that is tuned such that these elements compensate the intrinsic elements of $Y_{n}^{\prime}$ at the operating frequency $\omega_{\text {op }}$ with $Y_{n}^{\prime}\left(\omega_{\text {op }}\right)=0[5,16]$. The coupling admittance $Y_{n}^{\prime}$ thus is in resonance. Neglecting the stem impedance $L_{n}^{\prime}$, the resonance frequency is $\omega_{n}^{\prime}=$ $1 / \sqrt{L_{n}^{\prime \prime}} \sqrt{\left(1 / C_{n}^{\prime}+1 / C_{n}^{\prime \prime}\right)}$.

As has been pointed out in $[15,16]$, the longer the distance between post-couplers, the larger $C_{n}^{\prime}$, and the smaller $C_{n}^{\prime \prime}$ needs to be to add up to the series capacitance $C_{n}^{\prime}$ thus resulting in a larger post-coupler to drift tube distance $d_{n}$. This relationship is valid for comparisons between drift tube cells with varying length as well as DTL structures with varying post-coupler count, and explains variations in optimum post-coupler lengths analyzed, e.g., in [6] and [11]. Note that the condition $Y_{n}^{\prime}\left(\omega_{\mathrm{op}}\right)=0$ is not equivalent to the situation when the stem is tuned to its intrinsic resonance $\omega_{n}^{\prime \prime}=1 / \sqrt{L_{n}^{\prime \prime} C_{n}^{\prime \prime}}$ where $Y_{n}^{\prime}\left(\omega^{\prime \prime}\right)=\infty$. This resonance is used for parameter extraction described later.

Tilt-sensitivity measurements determine how much the accelerating field would be affected by tuning errors. In this measurement, the first or the last gap is perturbed artificially and a frequency shift is introduced that is compensated by a counterperturbation at the other end of the cavity. In [18], a variant to the classic tilt-sensitivity measurement has been introduced that is applied throughout this work and that has been shown to be fully equivalent to the traditional tilt-sensitivity measurement; The cavity is tuned by an average setting of the slug tuners to a frequency $\Delta \omega$ higher than the operating frequency $\omega_{\mathrm{op}}$ and then brought back to the operating frequency by a perturbation using a hollow tube at one end at a time, either at the first or the last gap. The hollow tube is then removed from the end and inserted at the beam pipe at the other end of the tank and the fields are measured again. The fields in the accelerating gaps are measured by a bead-pull system. All slug tuners are usually set at the same length. The difference in fields of each cell between the cases perturbed at the low-energy end and the high-energy end is the classical local tilt sensitivity. An excellent accuracy of the bead-pull system, well below $1 \%$ is essential in order to achieve the required stability and field flatness $[10,18,19]$.

\section{TS $_{\boldsymbol{n}}^{\prime}$ IN THE EQUIVALENT CIRCUIT}

In this article, the index le is used to describe the axial fields in the case that the first gap at the low-energy end is perturbed. In the same way he refers to the case of perturbing the high-energy end. The tilt sensitivity of the $n$th cell is defined as:

$$
\mathrm{TS}_{n}=\frac{2 \pi}{\Delta \omega}\left(E_{0 n}^{h e}-E_{0 n}^{l e}\right)
$$

where the $E_{0 n}^{h e}$ and $E_{0 n}^{l e}$ represent the normalized average accelerating electric fields at the $n$th gap with the corresponding perturbation. Note that it is essential that the perturbation measurements are undertaken at the same operating frequency, i.e., $\omega^{h e}=\omega^{l e}=\omega_{\mathrm{op}}$, and that the fields of both measurements are referred to the same stored energy.

Rewriting Kirchhoff's voltage law for the $n$th cell (3) for the case of low-energy end perturbation and the $(n+1)$ th cell for high-energy end perturbation, moving terms of $Y_{n}^{\prime}$ to the right-hand side, and adding these two equations yields:

$$
\begin{gathered}
Z_{n} I_{n}^{h e}+Z_{n+1} I_{n+1}^{l e}-\frac{I_{n-1}^{h e}-I_{n}^{h e}}{Y_{n-1}^{\prime}}+\frac{I_{n+1}^{l e}-I_{n+2}^{l e}}{Y_{n+1}^{\prime}} \\
=-\frac{I_{n}^{h e}-I_{n+1}^{h e}-I_{n}^{l e}+I_{n+1}^{l e}}{Y_{n}^{\prime}} .
\end{gathered}
$$

Consider for the following, that bead-perturbation measurements are taken for different penetration of the $n$th post-coupler and that moving post-couplers has negligible effect on $C_{n}$, and $L_{n}$, while $C_{n}^{\prime \prime}$ and thus the coupling admittance $Y_{n}^{\prime}$ is a function of $l_{n}^{\prime \prime}$. Since the frequency is kept constant at $\omega=\omega_{\mathrm{op}}$, perturbing the high-energy end changes $Z_{N}$ while the rest of the circuit elements do not change.

As can be derived recursively from the system of equations (3), regardless of $Y_{n}^{\prime}$, the current ratios $I_{n}^{\text {he }} / I_{1}^{\text {he }}$ and $I_{n-1}^{\text {he }} / I_{1}^{\text {he }}$ are independent of the perturbation. When the reference current $I_{1}^{\text {he }}$ is kept constant over all measurements, thus $Z_{n} I_{n}^{h e}-\left(I_{n-1}^{h e}-I_{n}^{h e}\right) / Y_{n-1}^{\prime}=$ const. In the same way, for a low-energy end perturbation, with $I_{N}^{l e}$ constant, also $Z_{n+1} I_{n+1}^{l e}-\left(I_{n+2}^{l e}-I_{n+1}^{l e}\right) / Y_{n+1}^{\prime}=$ const. The left-hand side of Eq. (5) therefore remains constant regardless of the $n$th post-coupler length and the numerator of the right-hand side becomes proportional to the admittance $Y_{n}^{\prime}$ :

$$
I_{n}^{l e}-I_{n}^{h e}-I_{n+1}^{l e}+I_{n+1}^{h e} \propto Y_{n}^{\prime} .
$$

Using the normalized average axial accelerating field $E_{0 n}=U_{n} / l_{n} / \bar{E}_{0}$ in the gap with the normalization $\bar{E}_{0}=U_{\text {tot }} / l_{\text {tot }}=\sum_{n} E_{0 n} l_{n} / \sum_{n} l_{n}$, the condition of a constant reference that is independent over all measurements is fulfilled with good approximation. Introducing this reference via the relation $I_{n}=j \omega C_{n} U_{n}$ in (6), and rewriting it in terms of tilt sensitivity $\mathrm{TS}_{n}(4)$ yields: 


$$
C_{n+1} l_{n+1} \mathrm{TS}_{n+1}-C_{n} l_{n} \mathrm{TS}_{n} \propto Y_{n}^{\prime} .
$$

With the stored energy $U_{n}^{2} C_{n} / 2$ approximately proportional to the cell length $l_{n}$, and with the accelerating gradient $E_{0 n}=U_{n} / l_{n} / \bar{E}_{0}$ constant over all cells of a DTL as usually the case, $C_{n} l_{n} \approx$ const over all cells. Simplifying (7) with this approximation gives:

$$
\mathrm{TS}_{n}^{\prime}=\mathrm{TS}_{n+1}-\mathrm{TS}_{n} \propto Y_{n}^{\prime} .
$$

The right-hand side when divided either by the length of the structure or the number of cells between the two TS values (here 1), can be understood as a discrete difference quotient of TS, and $\mathrm{TS}_{n}^{\prime}$ is thus the local tilt-sensitivity slope, here valid for structures where there is a post-coupler every drift tube. It is used in the following as the principal measure for stabilization.

Writing out $Y_{n}^{\prime}, \mathrm{TS}_{n}^{\prime}$ can now be analyzed in more detail:

$$
\mathrm{TS}_{n}^{\prime} \propto\left(j \omega C_{n}^{\prime}+\frac{1}{j \omega L_{n}^{\prime}}\right)+\frac{j \omega C_{n}^{\prime \prime}}{1-\omega^{2} L_{n}^{\prime \prime} C_{n}^{\prime \prime}} .
$$

Moving post-couplers has negligible effect on the electromagnetic field distribution in the rest of the cell. Therefore variation of $C_{n}^{\prime}$ and $L_{n}^{\prime}$ is negligible and since the resonance frequency is always kept at $\omega_{\mathrm{op}}$, the terms in parentheses are approximately constant. The inductance of the postcoupler $L_{n}^{\prime \prime}$ is considered approximately constant within the range of the variation of the post-coupler for stabilization.

The capacitance $C_{n}^{\prime \prime}$ of the post-coupler depends strongly on the distance $d_{n}=R_{c}-R_{d}-l_{n}^{\prime \prime}$ between the postcoupler tip and drift tube surface with $R_{c}$ and $R_{d}$ the cavity and drift tube radii, respectively. Since the postcoupler diameter is considerably smaller than the wavelength, one can apply a quasistatic approximation. In order to understand the qualitative behavior, the capacitance $C_{p}$ is approximated with a two plate capacitor $C_{n}^{\prime \prime}=\epsilon_{0} A / d_{n}$ with $A$ being an equivalent post-coupler tip area. Applying these approximations to Eq. (9) $\mathrm{TS}_{n}^{\prime}$ can be fitted by the following function:

$$
\mathrm{TS}_{n}^{\prime}=\alpha_{n}+\beta_{n} \frac{1}{l_{n}^{\prime \prime}-l_{n}^{r}}
$$

with the fitting parameters $\alpha_{n}$ and $\beta_{n}$, where $l_{n}^{\prime \prime}$ is the postcoupler length and $l_{n}^{r}=R_{c}-R_{d}-\epsilon_{0} A \omega^{2} L_{n}^{\prime \prime}$ is a reference length for the post-coupler.

\section{ANALYSIS OF TS ${ }_{n}^{\prime}$ IN 3D SIMULATIONS}

In this section, simulations with HFSS ${ }^{\mathrm{TM}}$ by Ansys Inc. are used to verify the behavior of $\mathrm{TS}_{n}^{\prime}$ for a single postcoupler and to study how electromagnetic fields change around the post-coupler as the post-coupler length varies. In order to avoid effects from cell mismatches in the simulations, a structure of 8 identical cells is simulated that is equipped with 7 post-couplers. The cell has general
Linac4 DTL dimensions and its length is $88 \mathrm{~mm}$. All postcouplers are initially set at $187 \mathrm{~mm}$. In all simulations, the electric field is normalized such that the total stored electromagnetic energy in the structure is $1 \mathrm{~J}$. Post-coupler 4 at the center of the structure is varied from $155 \mathrm{~mm}$ to $200 \mathrm{~mm}$ while other post-couplers are kept at $187 \mathrm{~mm}$. The frequency shift of $\mathrm{TM}_{010}$ and the variation of $\mathrm{TS}_{n}^{\prime}$ are plotted in Fig. 3.

Moving the post-coupler leads to a change in the $\mathrm{TM}_{010}$ modal resonance frequency. As is shown in the figure, the resonance frequency passes through a point of strong variation at a length of $l_{4}^{r}=170 \mathrm{~mm}$. Generally speaking, if the cavity is equipped with $N$ post-couplers, the postcoupler passband involves $N$ post-coupler modes. While the post-couplers are short, some post-coupler modes lie at a higher frequency than the $\mathrm{TM}_{010}$ mode. Increasing the post-coupler lengths, the post-coupler passband moves toward lower frequencies. As one of the post-coupler modes confluences with the $\mathrm{TM}_{010}$ mode, the $\mathrm{TM}_{010}$ frequency changes strongly. Further increasing the postcoupler length, the post-coupler mode goes to a lower frequency than the $\mathrm{TM}_{010}$ mode and the $\mathrm{TM}_{010}$ resonance frequency returns back to the original value without mode coupling.

In this example, the dependency of $\mathrm{TS}_{4}^{\prime}$ on the length of the respective post-coupler is depicted in the lower graph of
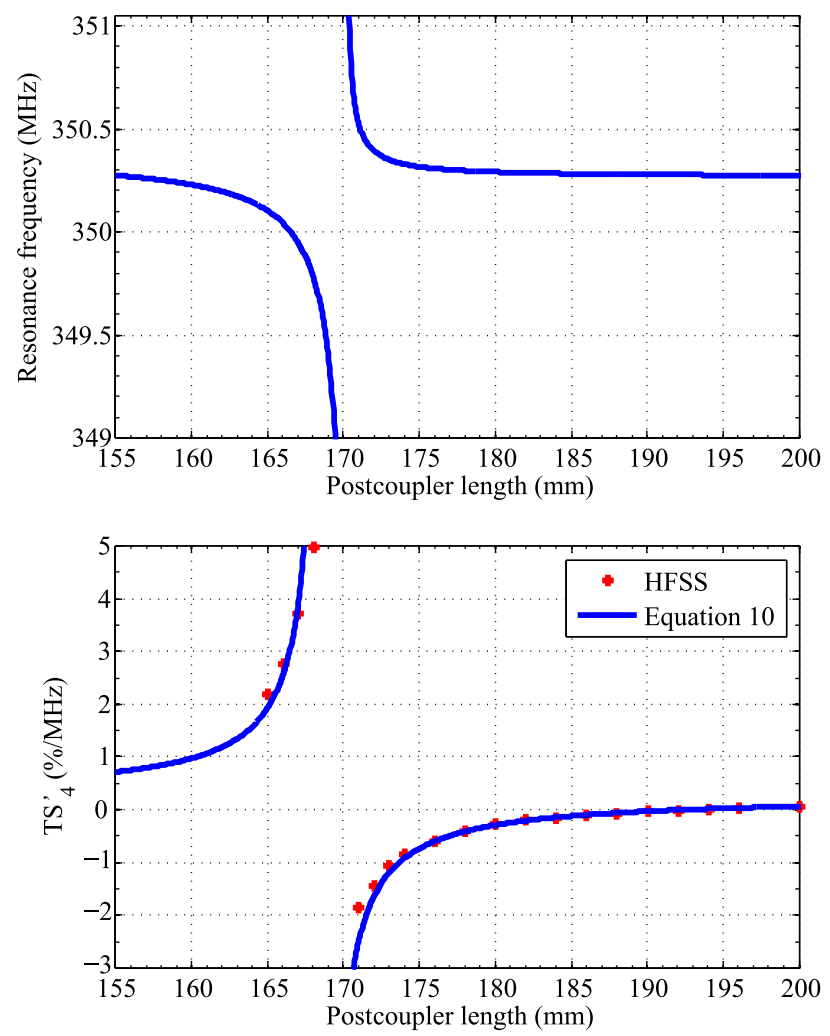

FIG. 3. Top: Variation of the resonance frequency of the $\mathrm{TM}_{010}$ mode as a function of the length of post-coupler $4 l_{4}^{\prime \prime}$. Bottom: Simulation of $\mathrm{TS}_{4}^{\prime}$ (dotted) fitted by equation (10). 
Fig. 3. Initially when the post-coupler 4 has a length of $155 \mathrm{~mm}, \mathrm{TS}_{4}^{\prime}$ has a positive value, and increasing the penetration increases it. At the same length $l_{4}^{r}$ as in the frequency curve, $\mathrm{TS}_{4}^{\prime}$ has a pole where it changes suddenly from positive to negative infinite values. With Eq. (9) this pole can be understood as the point where the inner resonance frequency of the post-coupler is tuned to the operating frequency $\omega_{\mathrm{op}}=1 / \sqrt{L_{n}^{\prime \prime} C_{n}^{\prime \prime}}$. As important as this finding is for the analysis, this resonance is not a point to be sought in operation.

It has been pointed out before that the objective of stabilization is the compensation of the intrinsic drift tube to wall elements by setting $Y_{n}^{\prime}\left(\omega_{\mathrm{op}}\right)=0$ at the operating frequency as this is what reduces the influence of tuning errors on neighboring cells. With the finding that $\mathrm{TS}_{n}^{\prime} \propto Y_{n}^{\prime}$, the target certainly is $\mathrm{TS}_{n}^{\prime}=0$. The optimum stabilization point in this example is around $187 \mathrm{~mm}$. The correspondence between frequency and $\mathrm{TS}_{n}^{\prime}$ measurements (Fig. 3) shows that $l_{n}^{r}$ can be determined from $\mathrm{TM}_{010}$ frequency shift measurements. It is then straightforward to extract the fitting parameters $\alpha_{n}$ and $\beta_{n}$ from $\mathrm{TS}_{n}^{\prime}$ measurements at two different post-coupler lengths. The correct post-coupler length is found as $l_{n}^{\prime \prime}=l_{n}^{r}-\beta_{n} / \alpha_{n}$. It goes without saying that the extraction of the fitting parameters is a step toward the extraction of the elements of the equivalent circuit. However it is not required to know the actual circuit elements in order to stabilize the structure.

In Fig. 4, the longitudinal axial plane view of the electric field for the 8 cell structure is plotted. The simulations are done for two different post-coupler lengths. In the first figure, post-coupler 4 is set at $l_{4}^{\prime \prime}=l_{4}^{r}=170 \mathrm{~mm}$ and in the bottom that post-coupler is set at the optimum length of $187 \mathrm{~mm}$. The same perturbation by a hollow tube at the left end of the structure is applied to both cases. As is apparent, when the distance $d_{n}$ between post-coupler and drift tube is at its optimum value, the electric field is concentrated almost entirely between cells. When a post-coupler is set to

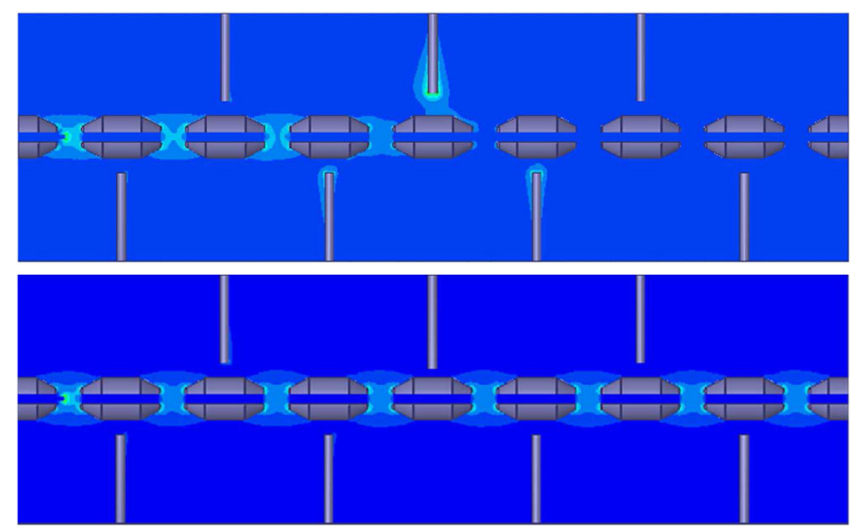

FIG. 4. Axial view of the electric field in an 8 equal-cell DTL perturbed at the left end. Bottom: All post-couplers are set at $187 \mathrm{~mm}$. Top: post-coupler 4 is set to its resonance length $l_{4}^{r}=$ $170 \mathrm{~mm}$ with the coupling admittance $Y_{4}^{\prime}=\infty$. its resonance length, as is shown for post-coupler 4, a considerable amount of electric field gets concentrated at that post-coupler.

From the plots one can deduce that fields between drift tubes on the right-hand side of the excited post-coupler 4 are close to zero and thus the voltage between the drift tube and the tank wall at the excited post-coupler is close to zero as well. In consequence, the coupling admittance must be $Y_{4}^{\prime}=\infty$ and the post-coupler thus is proved to be in resonance. One can also see in the plot that the two neighboring post-couplers are excited to some extent as well. The resonating system of post-coupler 4 is not independent of its neighbors as the current loop needs to be closed from one side of the tank wall to the other. The resonating current of post-coupler 4 is split equally on the neighboring post-couplers who participate in the resonance.

\section{MEASUREMENT OF TS ${ }_{n}^{\prime}$ DEPENDENCIES}

So far local tilt-sensitivity slope has been discussed on the basis of an equivalent-circuit model and HFSS simulations. In this section, the practical value of the new technique for the stabilization of DTL cavities is demonstrated using the CERN Linac4 DTL as an example. The Linac4 DTL consists of 3 cavities operating at $352.2 \mathrm{MHz}$ and at duty cycles of up to $10 \%$ for the acceleration of $\mathrm{H}^{-}$-ion beams of up to $40 \mathrm{~mA}$ average pulse current from 3 to $50 \mathrm{MeV}$. Tank 2 is a 7.34 meter long cavity and it contains 42 cells [20]. In this structure, cell length increases from 137 to $210 \mathrm{~mm}$. The post-couplers are distributed every two drift tubes and the 20 slug tuners are distributed horizontally along the axis. Tank 3 is a 7.25 meter long structure and contains 30 cells. The cell lengths increase from 219 to $263 \mathrm{~mm}$. All drift tubes are equipped with a post-coupler and 17 slug tuners are used to tune the electric field. The inner radius of the tanks $R_{c}$ is $260 \mathrm{~mm}$, and the drift tube radius $R_{d}$ is $45 \mathrm{~mm}$ resulting in a drift tube to tank distance of $215 \mathrm{~mm}$ to be considered as a hard limit for post-couplers [21].

As an example, the dependency of $\mathrm{TS}_{7}^{\prime}$ of post-coupler 7 of Tank 3 of the Linac4 DTL is investigated further. The reader shall note however that all post-couplers without exception show the same qualitative behavior and varying cell length is not an issue. In the measurements of $\mathrm{TS}_{7}^{\prime}$, the post-coupler length is varied between $155 \mathrm{~mm}$ and $196 \mathrm{~mm}$, while all other post-couplers are fixed at $186 \mathrm{~mm}$ (Fig. 5). The results show that Eq. (10) fits well the measurements. For post-coupler 7 the best stabilization is found at $l_{7}^{\prime \prime}=182 \mathrm{~mm}$. As in the simulations before, the $\mathrm{TM}_{010}$ modal resonance frequency has a strong variation at $l_{7}^{r}=170 \mathrm{~mm}$ as does the $\mathrm{TS}_{n}^{\prime}$ (Fig. 6). It is thus straightforward to determine $l_{n}^{r}$ by spectral measurements alone.

So far $\mathrm{TS}_{n}^{\prime}$ has been analyzed under the assumption that just one post-coupler is adjusted in length and that all other post-couplers remain at fixed length. Since the objective is 


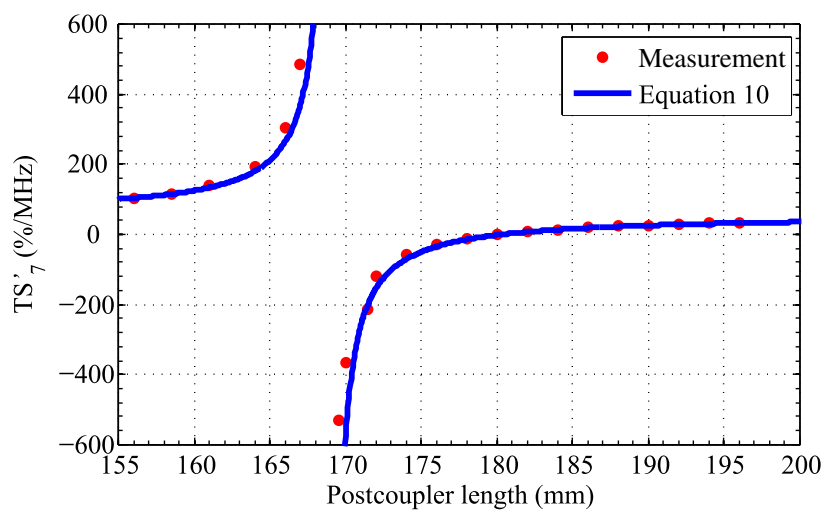

FIG. 5. The variation of $\mathrm{TS}_{7}^{\prime}$ as a function of the length of postcoupler $7 l_{7}^{\prime \prime}$. The behavior is well defined and can be fit using Eq. (10).

to stabilize the field all along the tank, the influence of neighboring post-couplers needs to be taken into account. The length of post-coupler 27 in Tank 3 has been measured while just one of its neighbors is adjusted (Fig. 7). Moving the next-neighbor post-couplers $l_{26}^{\prime \prime}$ or $l_{28}^{\prime \prime}$ changes the reference length for resonance $l_{27}^{r}$ strongly. This dependency on the next neighbors readily emerges from the $Y_{n-1}^{\prime}$ and $Y_{n+1}^{\prime}$ dependencies on the left-hand side of Eq. (5). The lengths of the second-next neighbors, i.e., post-couplers 25 and 29, $l_{25}^{\prime \prime}$ and $l_{29}^{\prime \prime}$ have almost no influence and $l_{27}^{r}$ varies very little regardless of the other post-coupler lengths. Changing the second-next neighbors post-coupler in (5) for example, which means varying $Y_{n-2}^{\prime}$, the axial field ratio $I_{n}^{h e} / I_{n+1}^{h e}$ does not change, and consequently $\mathrm{TS}_{n}^{\prime}$ remains unaffected. One can thus conclude that other post-couplers except the nearest neighbors do not affect $\mathrm{TS}_{n}^{\prime}$. This finding might be surprising in the light that mutual inductances have been neglected. Including these however in the circuit model and writing down the equations for $\mathrm{TS}_{n}^{\prime}$ shows that they are mostly canceling out. Taking advantage of the independency from second-next neighbor post-couplers, it

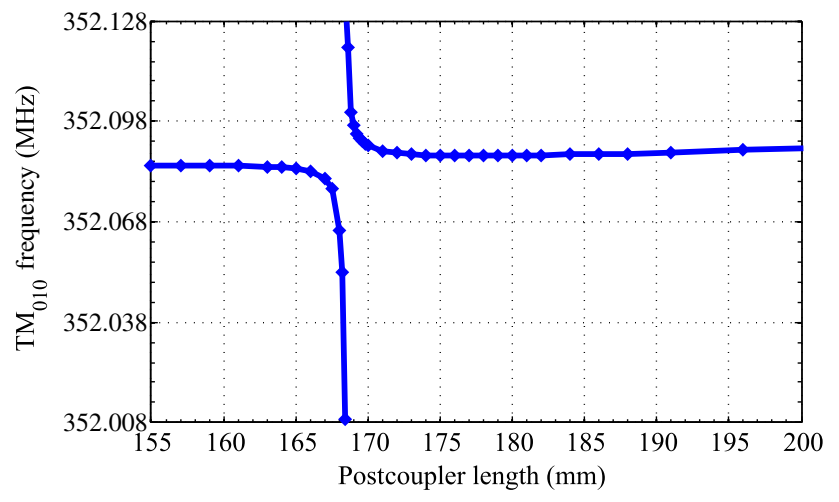

FIG. 6. Frequency shift of the $\mathrm{TM}_{010}$ mode as a function of the length of post-coupler $7 l_{7}^{\prime \prime}$. It is straightforward to measure $l_{n}^{r}$ accurately by varying the post-coupler length and observing the spectrum.

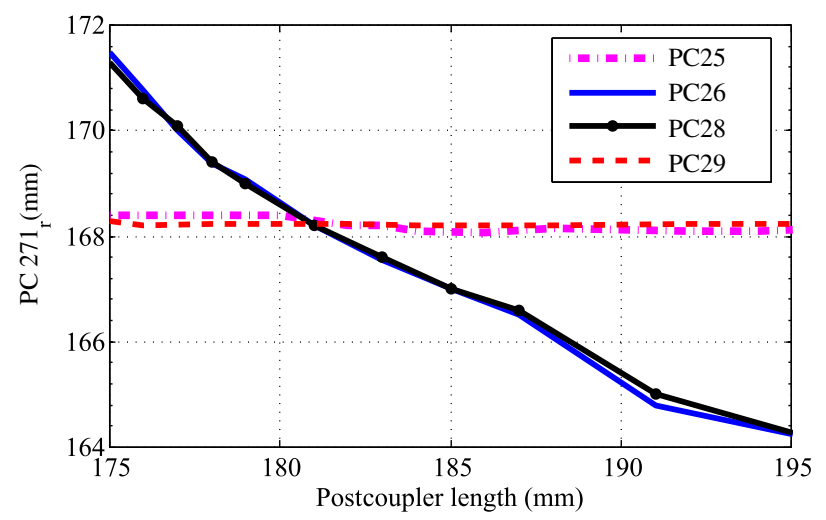

FIG. 7. The dependency of resonance length $l_{27}^{r}$ on the neighboring post-couplers. The nearest-neighbor post-couplers influence the resonance strongly but from the second nearest neighbors, post-couplers do not affect $l_{27}^{r}$. This important result allows for adjustment of every second post-coupler concurrently.

now becomes possible to adjust more than one post-coupler at the same time. This is a valuable ingredient to the stabilization procedure to be discussed later.

In the following, the influence of tuner movements on the tilt sensitivity is studied. From field simulations one can see that tuners directly influence the cell inductance $L_{n}$, but they have no direct effect on the coupling admittance or on the tilt sensitivity. In order to verify this finding, the tilt sensitivity is measured for different sets of tuner penetration (Fig. 8). For this analysis, the DTL structure is well stabilized at the operating frequency of $352.098 \mathrm{MHz}$ in air, and the first three measurements are taken at this resonance frequency. All tuners are set at the same length except for one that is used to adjust the frequency to the operating frequency. This measurement is repeated for three different tuners along the structure. Tuner 1 is located at the beginning, tuner 13 somewhere in the middle and tuner 17 at the end of the cavity. Each tuner is moved inwards by

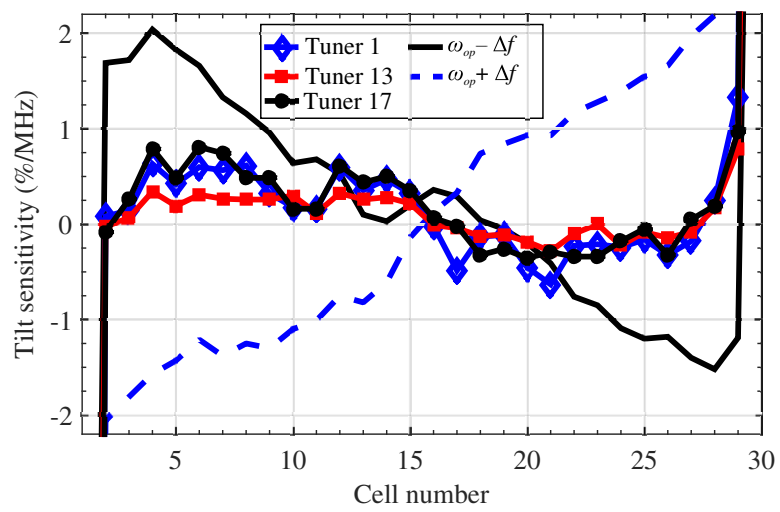

FIG. 8. Variation of the tilt sensitivity with individual adjustment of tuners 1,13 , and 17 . The influence by individual tuners is small, while there is a clear dependency on the resonance frequency of the structure when adjusting all tuners uniformly to an offset of $\pm 0.02 \mathrm{MHz}$. 
around half of its length $(80 \mathrm{~mm})$ so that the local perturbation is considerable. As is shown in the figure, the tilt sensitivity does not change when the structure resonance frequency remains constant.

In Fig. 8, also two cases are shown in which all tuners are moved uniformly from their initial length to a resonance frequency with $\pm 0.02 \mathrm{MHz}$ offset from the target frequency $\omega_{\mathrm{op}}$. The change in operating frequency leads to an overall slope in tilt sensitivity. $\mathrm{TS}_{n}^{\prime}$ is proportional to $Y_{n}^{\prime}$ and the change in operating frequency moves this element off the resonance point $Y_{n}^{\prime}=0$, and leads to small compensation currents that are similar in size on all post-couplers all over the cavity. In conclusion, the cavity needs to be stabilized at the operating frequency first and only then tuned for field flatness.

\section{STABILIZATION PROCEDURE}

As mentioned earlier, the goal of stabilization is to reduce the sensitivity of the axial accelerating field to perturbations at the operating frequency $\omega_{\mathrm{op}}$. It is thus essential that all measurements are made at the operating frequency. The tilt-measurement technique has been described at the end of Sec. II.

The stabilization procedure is: (1) Set all post-couplers to the same initial length such that the post-coupler passband approximately closes in confluence with the cavity mode passband as described in $[2,16,18]$. Note that this point is reached sufficiently well when the first post-coupler mode is as much in frequency below the $\mathrm{TM}_{010}$ mode as the $\mathrm{TM}_{011}$ mode is above. (2) Measure the tilt sensitivity and analyze $\mathrm{TS}_{n}^{\prime}$ for every cell. (3) Set all post-couplers of one cavity side at a new length. The post-couplers of the other cavity side must not change. (4) Measure the tilt sensitivity and analyze $\mathrm{TS}_{n}^{\prime}$. (5) Measure the resonance length $l_{n}^{r}$ of all these post-couplers. Move each post-coupler until the postcoupler mode is in full resonance with the $\mathrm{TM}_{010}$ mode. Return the post-coupler to the previous length and continue with the next post-coupler on the same side. (6) Calculate $\alpha_{n}$ and $\beta_{n}$ for each post-coupler from two tilt-sensitivity measurements [Eq. (10)]. The post-coupler length for $\mathrm{TS}_{n}^{\prime}=0$ is $l_{n}^{\prime \prime}=l_{n}^{r}-\beta_{n} / \alpha_{n}$ (Fig. 9). (7) Set all postcouplers to the calculated value and measure the tilt sensitivity again. (8) Repeat steps 3 to 7 for the postcouplers of the other cavity side in order to improve the tilt sensitivity further.

Note that the solution is not unique. As the procedure starts out with one cavity side and continues with the other, the post-coupler settings will not be fully symmetric (Fig. 10). Tilt-sensitivity measurements show that applying the procedure once on either side is sufficient and that a slight asymmetry in the post-coupler setting is not of particular importance. As can be seen from the equivalent-circuit model, the compensation of the drift tube to cavity capacitance $C_{n}^{\prime}$ by some fraction is just moved to the next post-coupler on the other cavity side. In case that a
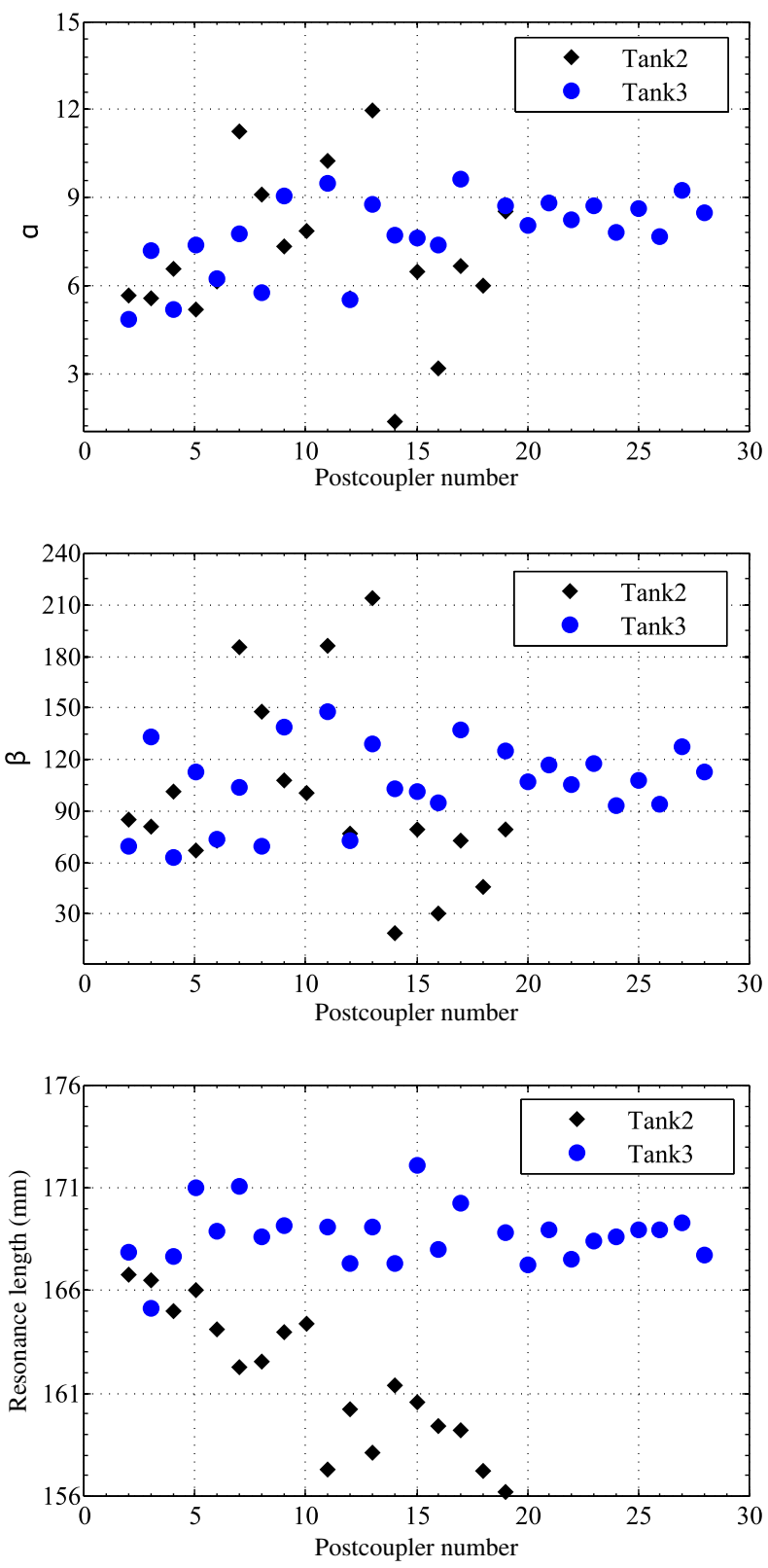

FIG. 9. Parameters $\alpha_{n}, \beta_{n}$, and the resonance length $l_{n}^{r}$ for all post-couplers of Tank 2 (diamonds) and Tank 3 (dots). Note that the values are measured separately on either side (even- or oddnumbered post-couplers) and that all parameters depend on the settings of the next-neighbor post-couplers.

more symmetric setting is sought, averaging of the postcoupler distances and a repetition of the procedure can be considered. In any case, starting out from a good initial post-coupler setting keeps asymmetries low.

The stabilization procedure by $\mathrm{rf}$ measurements requires the search of the resonance frequency $\omega^{\prime \prime}$ of individual postcouplers and fitting the $Y^{\prime}$ curve. In the case that one considers stabilization by 3D simulations however, searching the resonance of individual post-couplers is not as straightforward. Instead the use of a root-finding algorithm 
that defines the next setting for each post-coupler individually appears to be faster. Under the condition that reasonable starting values have been selected and all postcouplers on a cavity side are adjusted in parallel, only few simulation runs are required to find the optimum postcoupler settings.

So far, the stabilization procedure of drift tube cavities equipped with a post-coupler at every drift tube has been discussed. As is common practice at the low energy tanks of DTLs however, a post-coupler only every two or three drift tubes is used where the drift tubes are closely spaced for two reasons: First the drift tube spacing might not allow for more post-couplers and, second, roughly speaking each post-coupler is capable of compensating a certain drift tube to cavity capacitance $C_{n}^{\prime}$ independent of the number of drift tubes per unit-length. In this case, the definition of $\mathrm{TS}_{n}^{\prime}$ needs to be modified. With $R$ being the number of cells per post-coupler and the $m$ th cell located before the $n$th post-coupler, the general difference in tilt sensitivity of the $n$th post-coupler is defined as:

$$
\mathrm{TS}_{n}^{\prime}=\frac{\mathrm{TS}_{m+R}-\mathrm{TS}_{m}}{R}
$$

The generalized local tilt-sensitivity slope $\mathrm{TS}_{n}^{\prime}$ is thus the tilt-sensitivity slope between successive cells with postcouplers. The same stabilization procedure applies to this modified definition.

\section{TUNING PROCEDURE}

So far post-couplers have been adjusted to the optimum length, bringing the coupling admittance between cells close to zero. The propagation of local cell errors is strongly suppressed and the axial field gets flat. However stabilization does not eliminate tuning errors of individual cells. An error in cell resonance frequency remains and translates into an error in the axial field amplitude of the individual cell. This field error can be compensated by an individual adjustment of slug tuners. At the same time slug tuners need to tune the cavity to the operating frequency $\omega_{\mathrm{op}}$. As the effect of tuners on fields and frequency of individual slug tuners are linearly independent of each other, they can be superposed, and the optimum tuner lengths are found as the solution to a constrained linear least squares problem.

The tuning procedure is: (1) Adjust all tuners to the same initial length at the operating frequency $\omega_{\mathrm{op}}$, and measure the initial normalized axial electric fields $E_{0 i}^{i n}$ in all gaps $i$. (2) Move all tuners out by the same small amount in order to set the tank to a lower base frequency. (3) For all tuners individually, move each tuner $j$ in once, until the cavity resonates again at the operating frequency $\omega_{\mathrm{op}}$, and measure the normalized axial electric fields $E_{0 j, i}$ of all gaps $i$ and the corresponding tuner displacement $t_{j}$. (4) Calculate the transposed tuner sensitivity matrix $\mathrm{T}^{T}$ and the transposed target vector $B^{T}$ for all $N$ gaps and all $N_{T}$ tuners.
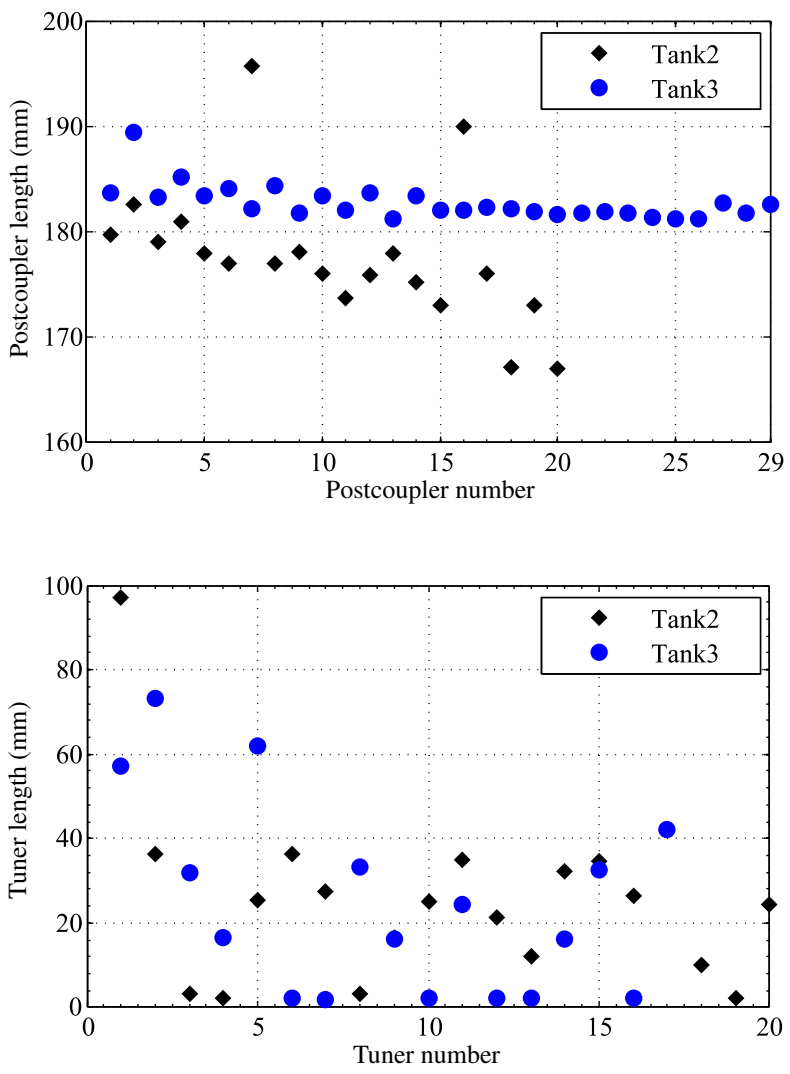

FIG. 10. Top: post-coupler lengths, bottom: tuner lengths of Tank 2 (diamonds) and Tank 3 (dots). The two post-couplers with exceptional over-length are due to an additional tuner piece that had been installed on these post-couplers for the compensation of the influence of the power-coupler.

$$
\begin{aligned}
\mathrm{T}_{i, j}^{T} & =\frac{E_{0 i, j}-E_{0 i}^{i n}}{t_{j}} \quad i=1 \text { to } N, \quad j=1 \text { to } N_{T} \\
B_{i}^{T} & =E_{0 i}^{i n}-1 \quad i=1 \text { to } N
\end{aligned}
$$

(5) Solve the constrained least squares problem, and apply the resulting relative tuner lengths to the tuner variations of all tuners with respect to step 1.

The unconstrained least squares solution can be written down algebraically but may contain tuner lengths that are out of bounds. From this point, one can fix excessive tuner lengths to the limits one by one, remove the corresponding equation and recalculate the new algebraic solution. Alternatively as is done in this work, one can use an optimization algorithm where the design parameters are constrained by the tuner range (Fig. 10).

\section{STABILIZATION AND TUNING OF THE CERN LINAC4 DTL}

In this section, the stabilization and tuning of the CERN Linac4 DTL structures is discussed using the procedures of the previous sections. Figures 11 and 12 show the measured 

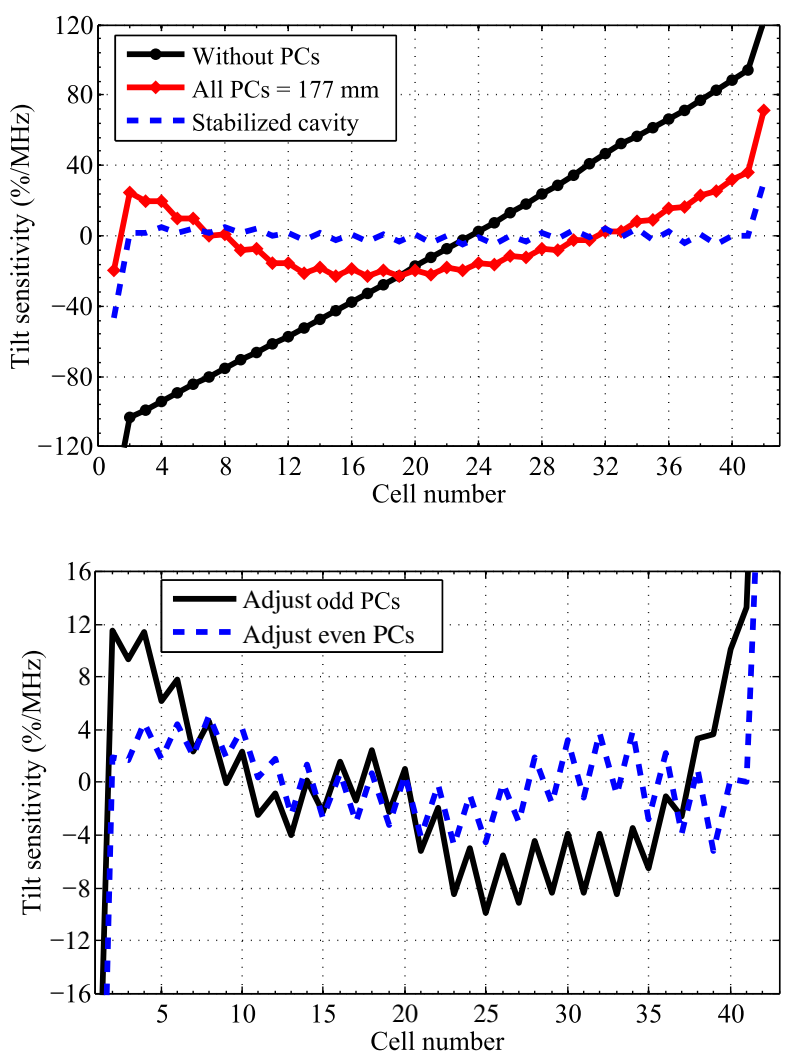

FIG. 11. Results of the post-coupler optimization for Linac4 Tank 2 . The initial tilt sensitivity is reduced by a factor of 20 . The jagged pattern is repeated in tilt sensitivity due to having only one post-coupler every two drift tubes. The lower graph is zoomed in tilt sensitivity.

tilt sensitivity of Tank 2 and Tank 3 during the stabilization process respectively. In Tank 3 , the initially measured tilt sensitivity is distributed between $\pm 100 \% / \mathrm{MHz}$. The end cell perturbation for the tilt-sensitivity measurements is $120 \mathrm{kHz}$. The global tilt sensitivity of the structure is reduced to $\pm 40 \% / \mathrm{MHz}$ by adjusting all post-couplers to the length of $181 \mathrm{~mm}$ (step 1). As is shown in Fig. 12, just with the evaluation of two tilt-sensitivity measurements (step 7), the odd-numbered post-couplers are adjusted and the tilt sensitivity is reduced to $\pm 3 \% / \mathrm{MHz}$. Repeating steps 3 to 7 , the even-numbered post-couplers are adjusted. The final tilt sensitivity remains within $\pm 1 \% / \mathrm{MHz}$ in all cells.

The results of stabilization of Tank 2 are plotted in Fig. 11. Since the structure has one post-coupler per two drift tubes, the general form of Eq. (11) is used with $R=2$ to calculate the $\mathrm{TS}_{n}^{\prime}$ values. First by adjusting the postcoupler band for confluence at a common post-coupler length of $177 \mathrm{~mm}$, the tilt sensitivity reduces from $\pm 100 \% / \mathrm{MHz}$ to $\pm 40 \% / \mathrm{MHz}$ (step 1 ). Then by adjusting the odd-numbered post-couplers, the tilt sensitivity reduces to $\pm 12 \% / \mathrm{MHz}$ and adjusting the even-numbered postcouplers, the tilt sensitivity becomes less than $\pm 5 \% / \mathrm{MHz}$. The absolute stability improvement in Tank 2 is less than on
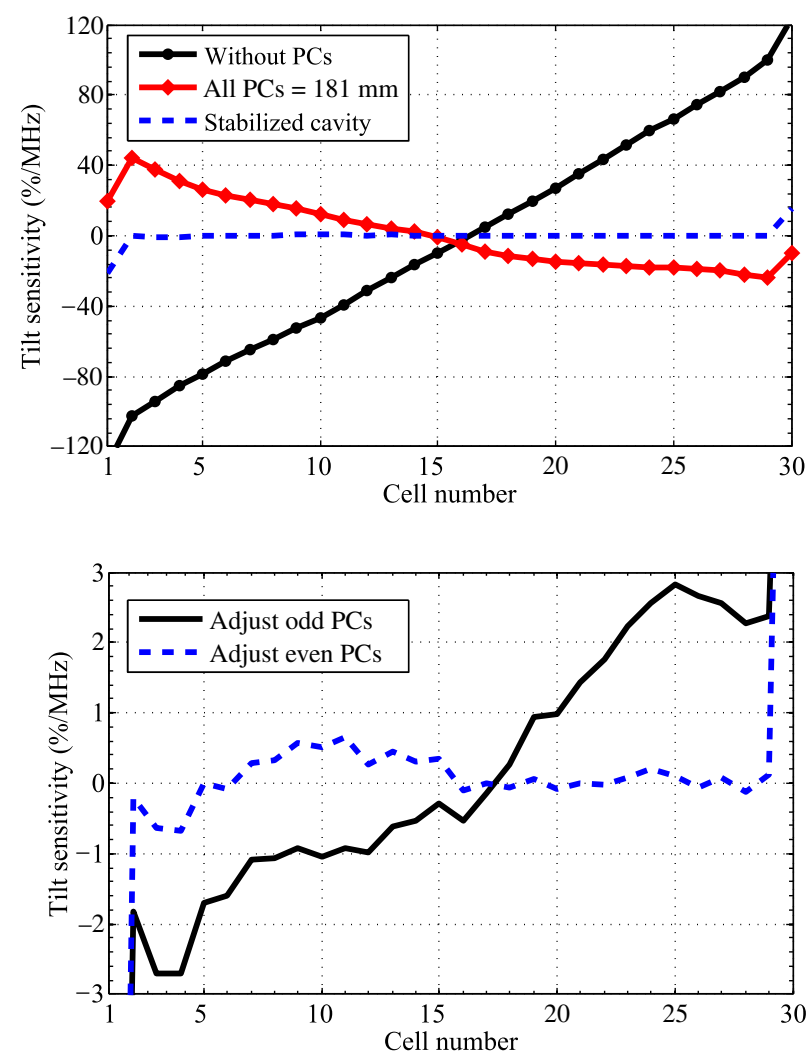

FIG. 12. Results of the post-coupler optimization for the Linac4 DTL Tank 3. The initial tilt sensitivity is reduced by a factor of 100. The lower graph is zoomed in tilt sensitivity and also shows the intermediate stabilization step of the adjustment of oddnumbered post-couplers.

Tank 3 as there is only one post-coupler every two drift tubes in Tank 2.

The parameters of the stabilization procedure $\alpha, \beta$, and $L_{n}^{r}$, and the final post-coupler lengths are shown in Figs. 9 and 10, respectively. As mentioned earlier, there is a slight asymmetry between the settings of post-couplers of the two sides which is considered acceptable. As pointed out earlier, the fact that post-couplers are closer to each other in Tank 3 than in Tank 2 makes that the capacitance $C_{n}^{\prime}$ is smaller there and that the post-coupler needs to provide a larger capacitance that is achieved with a shorter postcoupler to drift tube distance. Also note that the smaller this capacitance is, the larger need to be the differences in length to achieve the same absolute variation in capacitance. On Tank 2, post-couplers 7 and 16 are longer since they have a tuning ring that helps compensating the strong influence of the power coupler.

For the tuning of Tank 2 and Tank 3, the frequency has been lowered by $40 \mathrm{kHz}$ in step 2 of the tuning procedure. The corresponding tuner length is $31 \mathrm{~mm}$ in Tank 2 and $23 \mathrm{~mm}$ in Tank 3 . The tuner sensitivity matrix has been measured according to the tuning procedure described in Sec. VII. For both Tank 2 and Tank 3, the least squares 

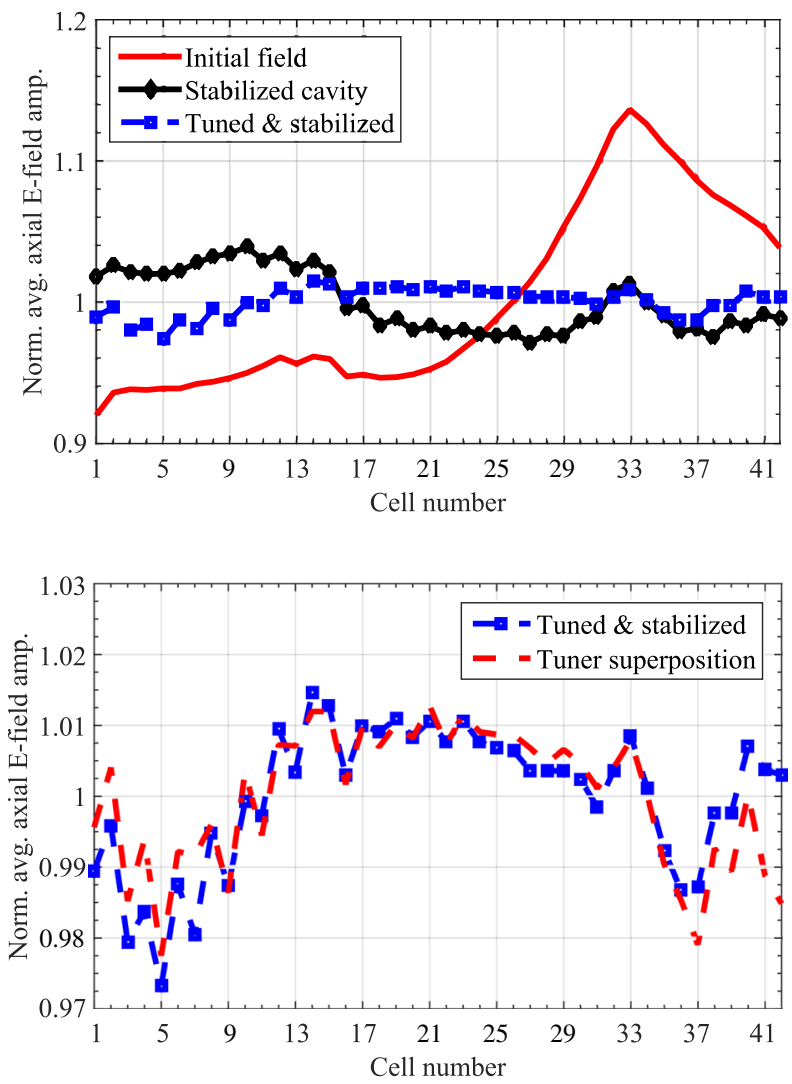

FIG. 13. Top: Field flatness in the Linac4 DTL Tank 2 before stabilization, after stabilization, and after tuning. Bottom: Zoom showing the final field flatness and the prediction from individual tuner measurements.

algebraic solution returns tuner lengths that are out of bounds. The reason for this is to be sought in the strong influence of the power couplers that cannot be fully compensated by the available tuner range. Instead an optimization algorithm that starts out with random sets of tuner lengths has been used. Applying optimization algorithms to this problem is straightforward as it allows limiting the design variable space, fixing lengths for some premanufactured pieces like those that are later replaced by movable tuners.

The field flatness has been brought to within $\pm 2 \%$ as required by beam physics [22], and the resonance frequency is set to $352.098 \mathrm{MHz}$ in air. In order to ease the final machining adjustment, post-coupler tips without tabs have been used. Tabs provide a larger tuning range and were considered as an option in case that tuning by slug tuners would not have been sufficient. Figure 10 shows the final tuner lengths, and Figs. 13 and 14 show the field of Tank 2 and Tank 3, respectively, before stabilization, after stabilization, and after tuning. The two peaks in the initial field of both cavities show the tuning error that is introduced by the power couplers. After stabilization but before tuning, the peaks in field flatness are already reduced significantly. After tuning, the field in Tank 3 is
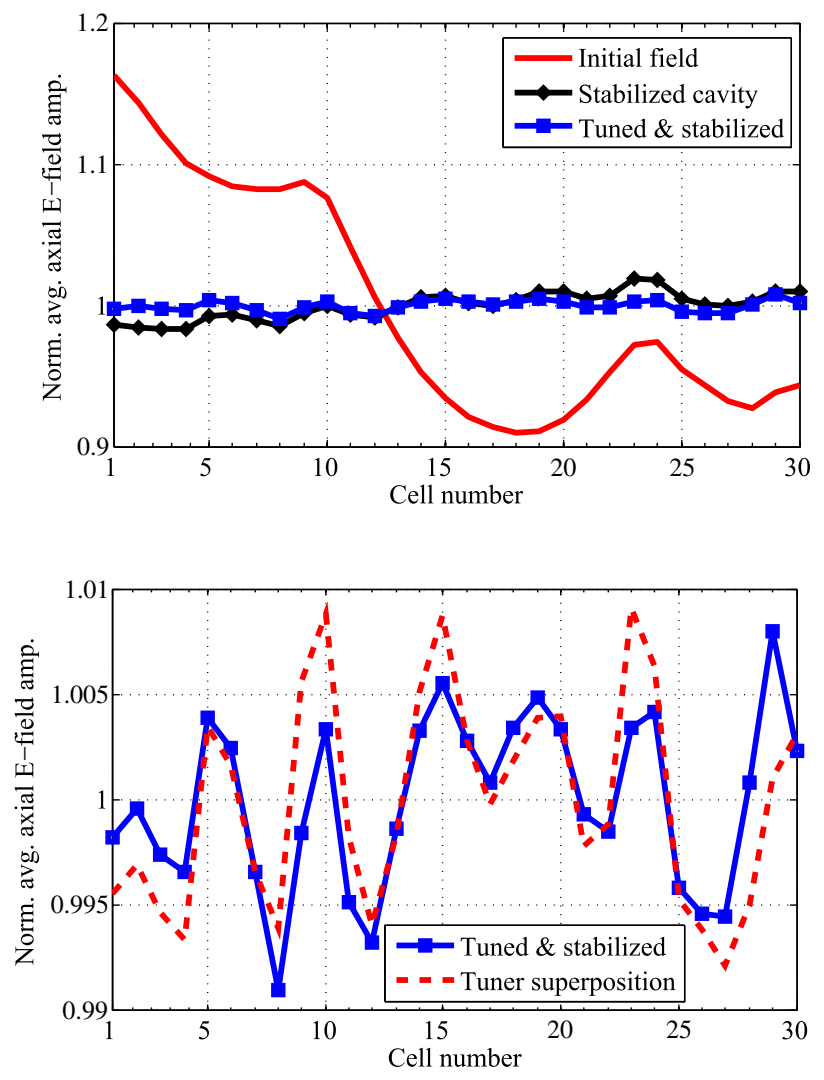

FIG. 14. Top: Field flatness Linac4 DTL Tank 3 before stabilization, after stabilization, and after tuning. Bottom: Zoom showing the final field flatness and the prediction from individual tuner measurements.

flatter than in Tank 2, due to the higher number in postcouplers that limit error propagation. As is shown in the zoomed graphs, the superposition of individual tuner effects comes very close to the final field measurement and confirms the tuning technique.

\section{CONCLUSIONS}

A straightforward and accurate technique for the stabilization and tuning of DTL tanks has been presented. The criterion of local tilt-sensitivity slope is established as the principal measure for stabilization and it is shown to be proportional to the coupling admittance. The behavior of the local tilt-sensitivity slope is analyzed by 3D HFSS simulations and structure measurements and as an outcome the procedure for tank stabilization is derived. The technique is considerably faster than the tedious iteration approach that has so far been applied to DTL stabilization in that it requires essentially only 4 tilt-sensitivity measurements independent of the structure length, and it provides accurate results. The application of the procedures has been demonstrated successfully on CERN's Linac4 DTL structures Tank 2 and Tank 3. 
[1] A. E. Knapp, D. A. Swenson, and J. M. Potter, Method and device for stabilization of the field distribution in drift tube linac, (1970), US Patent 3,501,734.

[2] D. A. Swenson, E. A. Meyer, J. M. Potter, and E. J. Schneider, in Proceedings of 6th International Conference on High-Energy Accelerators, Cambridge, Massachusetts, USA (Cambridge Electron Accelerator, 1967), pp. 167-173.

[3] J. Ungrin, S. Schriber, and A. Vokes, IEEE Trans. Nucl. Sci. 30, 3013 (1983).

[4] M. Sawamura and H. Takekoshi, Bull. Inst. Chem. Res. Kyoto Univ. 63, 9 (1985).

[5] S. Machida, T. Kato, and S. Fukumoto, IEEE Trans. Nucl. Sci. 32, 3259 (1985).

[6] F. Naito, T. Kato, E. Takasaki, and Y. Yamazaki, Part. Accel. 32, 1469 (1990).

[7] J. V. Bylinsky, S. K. Esin, A. V. Feschenko, P. N. Ostroumov, and S.Z. Zharylkapov, in Proceedings of 1988 Linear Accelerator Conference, Williamsburg, Virginia, USA (1988), pp. 158-160.

[8] F. Naito, H. Tanaka, M. Ikegami, T. Kato, E. Takasaki, and T. Ito, in Proceedings of Particle Accelerator Conference, PAC2003, Portland, Oregon, USA (IEEE, New York, 2003), pp. 2835-2837.

[9] T. Kato and S. Machida, in Proceedings of 1986 Linear Accelerator Conference, Stanford, California, USA (1986), pp. 119-121.

[10] J. H. Billen and A. H. Shapiro, in Proceedings of 1988 Linear Accelerator Conference, Williamsburg, Virginia, USA (1988), pp. 131-133.

[11] J. H. Billen, G. Spalek, and A. H. Shapiro, in Proceedings of 1988 Linear Accelerator Conference, Williamsburg, Virginia, USA (1988), pp. 125-127.
[12] A. Chao and M. Tigner, Handbook of Accelerator Physics and Engineering, 3rd ed. (Word Scientific, Singapore, 2006).

[13] P. M. Lapostolle and A. L. Septier, Linear Accelerators (North-Holland Publishing Company, Amsterdam, 1970).

[14] F. Grespan, G. De Michele, S. Ramberger, and M. Vretenar, in Proceedings of 25th International Linear Accelerator Conference, LINAC2010, Tsukuba, Japan (KEK, Tsukuba, Japan, 2011), pp. 578-580.

[15] F. Grespan, G. De Michele, S. Ramberger, and M. Vretenar, CERN Report No. CERN-sLHC-Project-Note0014, 2010.

[16] F. Grespan, Phys. Rev. ST Accel. Beams 15, 010101 (2012).

[17] T. P. Wangler, RF Linear Accelerators, 2nd ed. (WileyVCH, New York, 2008).

[18] M. R. Khalvati and S. Ramberger, in Proceedings of 27th Linear Accelerator Conference, LINAC2014, Geneva, Switzerland (2014), pp. 631-633.

[19] T. G. Jurgens, Report No. FERMILAB-LU-159, 1990.

[20] S. Ramberger, P. Bourquin, A. Cherif, Y. Cuvet, A. Dallocchio, G. Favre, J.-F. Fuchs, J.-M. Geisser, F. Gerigk, J.-M. Giguet, J. Hansen, M. Polini, S. Sgobba, N. Thaus, and M. Vretenar, in Proceedings of 27th Linear Accelerator Conference, LINAC2014, Geneva, Switzerland (2014), pp. 923-925.

[21] S. Ramberger, P. Bourquin, Y. Cuvet, A. Dallocchio, G. De Michele, F. Gerigk, J. M. Giguet, J. B. Lallement, A. M. Lombardi, E. Sargsyan, and M. Vretenar, in Proceedings of 25th International Linear Accelerator Conference, LINAC2010, Tsukuba, Japan (KEK, 2011), pp. 560-562.

[22] G. Bellodi, M. Eshraqi, M. Garcia Tudela, L. Hein, J. B. Lallement, S. Lanzone, A. M. Lombardi, P. Posocco, and E. Sargsyan, CERN Report, 2011. 